\title{
Representations of degenerate poly-Bernoulli polynomials
}

\author{
Taekyun Kim ${ }^{1,2}$, Dae San Kim, Jongkyum Kwon ${ }^{4 *}$ and Hyunseok Lee ${ }^{2}$
}

"Correspondence:

mathkjk26@gnu.ac.kr

${ }^{4}$ Department of Mathematics

Education and ERI, Gyeongsang

National University, Jinju 660-701, Republic of Korea

Full list of author information is

available at the end of the article

\begin{abstract}
As is well known, poly-Bernoulli polynomials are defined in terms of polylogarithm functions. Recently, as degenerate versions of such functions and polynomials, degenerate polylogarithm functions were introduced and degenerate poly-Bernoulli polynomials were defined by means of the degenerate polylogarithm functions, and some of their properties were investigated. The aim of this paper is to further study some properties of the degenerate poly-Bernoulli polynomials by using three formulas coming from the recently developed ' $\lambda$-umbral calculus'. In more detail, among other things, we represent the degenerate poly-Bernoulli polynomials by higher-order degenerate Bernoulli polynomials and by higher-order degenerate derangement polynomials.
\end{abstract}

Keywords: Degenerate poly-Bernoulli polynomials; Degenerate derangement polynomials; $\lambda$-umbral calculus

\section{Introduction}

Carlitz is the first one who initiated the study of degenerate versions of some special numbers and polynomials, namely the degenerate Bernoulli and Euler polynomials and numbers (see [2]). In recent years, studying degenerate versions of some special numbers and polynomials regained interests of some mathematicians with their interests not only in combinatorial and arithmetic properties but also in applications to differential equations, identities of symmetry, and probability theory (see $[9,10,12-14,17,19,21]$ and the references therein). It is noteworthy that studying degenerate versions is not only limited to polynomials but also can be extended to transcendental functions like gamma functions (see [13]).

The Rota's theory of umbral calculus is based on linear functionals and differential operators (see [3-7, 20, 23-27]). The Sheffer sequences occupy the central position in the theory and are characterized by the generating functions where the usual exponential function enters. The motivation for the paper [10] starts from the question that what if the usual exponential function is replaced with the degenerate exponential functions (see (2)). As it turns out, it corresponds to replacing the linear functional with the family of $\lambda$ linear functionals (see (12)) and the differential operator with the family of $\lambda$-differential operators (see (14)). Indeed, these replacements lead us to defining $\lambda$-Sheffer polynomials and degenerate Sheffer polynomials (see (16)).

(c) The Author(s) 2021. This article is licensed under a Creative Commons Attribution 4.0 International License, which permits use sharing, adaptation, distribution and reproduction in any medium or format, as long as you give appropriate credit to the original author(s) and the source, provide a link to the Creative Commons licence, and indicate if changes were made. The images or other third party material in this article are included in the article's Creative Commons licence, unless indicated otherwise in a credit line to the material. If material is not included in the article's Creative Commons licence and your intended use is not permitted by statutory regulation or exceeds the permitted use, you will need to obtain permission directly from the copyright holder. To view a copy of this licence, visit http://creativecommons.org/licenses/by/4.0/. 
As is well known, poly-Bernoulli polynomials are defined in terms of polylogarithm functions. Recently, as degenerate versions of such functions and polynomials, degenerate polylogarithm functions were introduced and degenerate poly-Bernoulli polynomials were defined by means of the degenerate polylogarithm functions, and some properties of the degenerate poly-Bernoulli polynomials were investigated (see [17]).

The aim of this paper is to further study the degenerate poly-Bernoulli polynomials, which is a $\lambda$-Sheffer sequence and hence a degenerate Sheffer sequence, by using the above-mentioned $\lambda$-linear functionals and $\lambda$-differential operators. In more detail, these polynomials are investigated by three different tools, namely a formula about representing a $\lambda$-Sheffer sequence by another (see (19)), a formula coming from the generating functions of $\lambda$-Sheffer sequences (see Theorem 1 ), and a formula arising from the definitions for $\lambda$-Sheffer sequences (see Theorems 6, 7). Then, among other things, we represent the degenerate poly-Bernoulli polynomials by higher-order degenerate Bernoulli polynomials and by higher-order degenerate derangement polynomials. The rest of this section is devoted to recalling the necessary facts that are needed throughout the paper, which includes the ' $\lambda$-umbral calculus'.

For $k \in \mathbb{Z}$ and $0 \neq \lambda \in \mathbb{R}$, the degenerate polylogarithm functions are defined by

$$
\operatorname{Li}_{k, \lambda}(x)=\sum_{n=1}^{\infty} \frac{(-\lambda)^{n-1}(1)_{n, 1 / \lambda}}{(n-1) ! n^{k}} x^{n} \quad(\text { see }[9,17]),
$$

where $(x)_{0, \lambda}=1,(x)_{n, \lambda}=x(x-\lambda) \cdots(x-(n-1) \lambda),(n \geq 1)$.

For any $\lambda \in \mathbb{R}$, the degenerate exponential functions are given by

$$
e_{\lambda}^{x}(t)=\sum_{n=0}^{\infty} \frac{(x)_{n, \lambda}}{n !} t^{n}, \quad e_{\lambda}(t)=e_{\lambda}^{1}(t)=\sum_{n=0}^{\infty} \frac{(1)_{n, \lambda}}{n !} t^{n} \quad(\text { see }[9,13]) .
$$

Note here that, for $\lambda \neq 0, e_{\lambda}^{x}(t)=(1+\lambda t)^{\frac{x}{\lambda}}$.

The compositional inverse $\log _{\lambda}(t)$ of $e_{\lambda}(t)$ is given by

$$
\log _{\lambda}(t)=\sum_{n=1}^{\infty} \frac{\lambda^{n-1}}{n !}(1)_{n, 1 / \lambda}(t-1)^{n}, \quad(\text { see }[9,13])
$$

From (1) and (3), we have

$$
\operatorname{Li}_{1, \lambda}(x)=-\log _{\lambda}(1-x) \quad \text { and } \quad \lim _{\lambda \rightarrow 0} \mathrm{Li}_{k, \lambda}(x)=\mathrm{Li}_{k}(x),
$$

where $\mathrm{Li}_{k}(x)$ are the polylogarithm functions defined by

$$
\operatorname{Li}_{k}(x)=\sum_{n=1}^{\infty} \frac{x^{n}}{n^{k}} \quad(|x|<1),(\text { see }[1,8,9,22]) .
$$

In [9], the degenerate poly-Bernoulli polynomials are defined by Kim-Kim as

$$
\frac{\operatorname{Li}_{k, \lambda}\left(1-e_{\lambda}(-t)\right)}{e_{\lambda}(t)-1} e_{\lambda}^{x}(t)=\sum_{n=0}^{\infty} B_{n, \lambda}^{(k)}(x) \frac{t^{n}}{n !} .
$$

When $x=0, B_{n, \lambda}^{(k)}=B_{n, \lambda}^{(k)}(0)$ are called the degenerate poly-Bernoulli numbers. 
It is well known that Carlitz's degenerate Bernoulli polynomials of order $r$ are defined by

$$
\left(\frac{t}{e_{\lambda}(t)-1}\right)^{r} e_{\lambda}^{x}(t)=\sum_{n=0}^{\infty} \beta_{n, \lambda}^{(r)}(x) \frac{t^{n}}{n !} \quad(\text { see }[2])
$$

For $r=1, \beta_{n, \lambda}^{(1)}(x)=\beta_{n, \lambda}(x)$ are called the degenerate Bernoulli polynomials.

From (5), we note that

$$
\sum_{n=0}^{\infty} B_{n, \lambda}^{(1)}(x) \frac{t^{n}}{n !}=\frac{\operatorname{Li}_{1, \lambda}\left(1-e_{\lambda}(-t)\right)}{e_{\lambda}(t)-1}=\frac{t}{e_{\lambda}(t)-1} e_{\lambda}^{x}(t)=\sum_{n=0}^{\infty} \beta_{n, \lambda}(x) \frac{t^{n}}{n !}
$$

By (7), we get $B_{n, \lambda}^{(1)}(x)=\beta_{n, \lambda}(x),(n \geq 0)$.

The degenerate Stirling numbers of the second kind appear as the coefficients in the expansion

$$
(x)_{n, \lambda}=\sum_{l=0}^{n} S_{2, \lambda}(n, l)(x)_{l} \quad(n \geq 0),(\text { see }[9,11])
$$

As the inversion formula of (8), the degenerate Stirling numbers of first kind appear as the coefficients in the expansion

$$
(x)_{n}=\sum_{l=0}^{n} S_{1, \lambda}(n, l)(x)_{l, \lambda} \quad(n \geq 0),(\text { see }[9]) .
$$

Thus, by (8) and (9), we have

$$
\frac{1}{k !}\left(e_{\lambda}(t)-1\right)^{k}=\sum_{n=k}^{\infty} S_{2, \lambda}(n, k) \frac{t^{n}}{n !}
$$

and

$$
\frac{1}{k !}\left(\log _{\lambda}(1+t)\right)^{k}=\sum_{n=k}^{\infty} S_{1, \lambda}(n, k) \frac{t^{n}}{n !} \quad(k \geq 0), \text { (see [9]). }
$$

In view of (6), the degenerate derangement polynomials of order $r(\in \mathbb{N})$ are defined by

$$
\frac{1}{(1-t)^{r}} e_{\lambda}^{-1}(t) e_{\lambda}^{x}(t)=\sum_{n=0}^{\infty} d_{n, \lambda}^{(r)}(x) \frac{t^{n}}{n !} \quad(\text { see }[12,19])
$$

When $r=1, d_{n, \lambda}(x)=d_{n, \lambda}^{(1)}(x)$ are called the degenerate derangement polynomials.

Note that $\lim _{\lambda \rightarrow 0} d_{n, \lambda}(x)=d_{n}(x)$, where $d_{n}(x)$ are the derangement polynomials and $d_{n}=$ $d_{n}(0)$ are the derangement numbers (see $[12,15,16,18]$ ).

We remark that the umbral calculus has long been studied by many people (see [3$7,20,23-27])$. For the rest of this section, we recall the necessary facts on the $\lambda$-linear functionals, $\lambda$-differential operators, $\lambda$-Sheffer sequences, and so on. The details on these can be found in the recent paper [10]. 
Let $\mathbb{C}$ be the field of complex numbers,

$$
\mathcal{F}=\left\{f(t)=\sum_{k=0}^{\infty} a_{k} \frac{t^{k}}{k !} \mid a_{k} \in \mathbb{C}\right\},
$$

and let

$$
\mathbb{P}=\mathbb{C}[x]=\left\{\sum_{i=0}^{\infty} a_{i} x^{i} \mid a_{i} \in \mathbb{C} \text { with } a_{i}=0 \text { for all but finite number of } i\right\} .
$$

For $f(t) \in \mathcal{F}$ with $f(t)=\sum_{k=0}^{\infty} a_{k} \frac{t^{k}}{k !}$ and $\lambda \in \mathbb{R}$, the $\lambda$-linear functional $\langle f(t) \mid \cdot\rangle_{\lambda}$ on $\mathbb{P}$ is defined by

$$
\left\langle f(t) \mid(x)_{n, \lambda}\right\rangle_{\lambda}=a_{n} \quad(n \geq 0),(\text { see }[10]) .
$$

By (12), we get

$$
\left\langle t^{k} \mid(x)_{n, \lambda}\right\rangle_{\lambda}=n ! \delta_{n, k} \quad(n, k \geq 0),(\text { see }[10]),
$$

where $\delta_{n, k}$ is the Kronecker symbol.

The $\lambda$-differential operators on $\mathbb{P}$ are defined by

$$
\left(t^{k}\right)_{\lambda}(x)_{n, \lambda}= \begin{cases}(n)_{k}(x)_{n-k, \lambda}, & \text { if } 0 \leq k \leq n \\ 0, & \text { if } k>n\end{cases}
$$

For $f(t)=\sum_{k=0}^{\infty} a_{k} \frac{t^{k}}{k !} \in \mathcal{F}$, and by (14), we get

$$
\begin{aligned}
& (f(t))_{\lambda}(x)_{n, \lambda}=\sum_{k=0}^{n}\left(\begin{array}{l}
n \\
k
\end{array}\right) a_{k}(x)_{n-k, \lambda} \quad(n \geq 0), \\
& \left(e_{\lambda}^{y}(t)\right)_{\lambda}(x)_{n, \lambda}=(x+y)_{n, \lambda} \quad(n \geq 0),(\text { see }[10]) .
\end{aligned}
$$

Let $f(t)$ be a delta series, and let $g(t)$ be an invertible series. Then there exists a unique sequence $S_{n, \lambda}(x)\left(\operatorname{deg} S_{n, \lambda}(x)=n\right)$ of polynomials satisfying the orthogonality conditions

$$
\left\langle g(t)(f(t))^{k} \mid S_{n, \lambda}(x)\right\rangle_{\lambda}=n ! \delta_{n, k} \quad(n, k \geq 0),(\text { see }[10]) .
$$

Here, $S_{n, \lambda}(x)$ is called the $\lambda$-Sheffer sequence for $(g(t), f(t))$, which is denoted by $S_{n, \lambda}(x) \sim$ $(g(t), f(t))_{\lambda}$. The sequence $S_{n, \lambda}(x)$ is the $\lambda$-Sheffer sequence for $(g(t), f(t))$ if and only if

$$
\frac{1}{g(\bar{f}(t))} e_{\lambda}^{y}(\bar{f}(t))=\sum_{n=0}^{\infty} S_{n, \lambda}(y) \frac{t^{n}}{n !} \quad(\text { see }[10])
$$

for all $y \in \mathbb{C}$, where $\bar{f}(t)$ is the compositional inverse of $f(t)$ such that $f(\bar{f}(t))=\bar{f}(f(t))=t$.

Let $S_{n, \lambda}(x) \sim(g(t), f(t))_{\lambda}$. Then, from Theorem 16 of [10], we recall that

$$
(f(t))_{\lambda} S_{n, \lambda}(x)=n S_{n-1, \lambda}(x), \quad(n \geq 1) .
$$


For $S_{n, \lambda}(x) \sim(g(t), f(t))_{\lambda}, r_{n, \lambda}(x) \sim(h(t), l(t))_{\lambda}$, we have

$$
S_{n, \lambda}(x)=\sum_{k=0}^{n} C_{n, k} r_{k, \lambda}(x), \quad(n \geq 0),(\text { see }[10])
$$

where

$$
C_{n, k}=\frac{1}{k !}\left\langle\frac{h(\bar{f}(t))}{g(\bar{f}(t))}(l(\bar{f}(t)))^{k} \mid(x)_{n, \lambda}\right\rangle_{\lambda} .
$$

Finally, we note that $\lambda$-umbral calculus has some merit over umbral calculus when dealing with $\lambda$-Sheffer sequences. As one example, we illustrate this with the problem of representing the degenerate Bernoulli polynomial $\beta_{n, \lambda}(x)$ in terms of the degenerate falling factorials $(x)_{k, \lambda}$. As before, let $f(t)$ and $g(t)$ be respectively a delta series and an invertible series. First, we recall that $S_{n}(x)$ is Sheffer for $(g(t), f(t))$ denoted by $S_{n}(x) \sim(g(t), f(t))$ if and only if

$$
\frac{1}{g(\bar{f}(t))} e^{x \bar{f}(t)}=\sum_{n=0}^{\infty} S_{n}(x) \frac{t^{n}}{n !} \quad(\text { see }[24])
$$

Next, we recall that, for $S_{n}(x) \sim(g(t), f(t)), r_{n}(x) \sim(h(t), l(t))$, we have

$$
S_{n}(x)=\sum_{k=0}^{n} C_{n, k} r_{k}(x), \quad(n \geq 0),(\text { see }[24]),
$$

where

$$
C_{n, k}=\frac{1}{k !}\left\langle\frac{h(\bar{f}(t))}{g(\bar{f}(t))}(l(\bar{f}(t)))^{k} \mid x^{n}\right\rangle .
$$

Now, we observe that $\beta_{n, \lambda}(x)$ is $\lambda$-Sheffer for $\left(\frac{e_{\lambda}(t)-1}{t}, t\right), \beta_{n, \lambda}(x) \sim\left(\frac{e_{\lambda}(t)-1}{t}, t\right)_{\lambda}$ and Sheffer for $\left(\frac{\lambda\left(e^{t}-1\right)}{e^{\lambda t}-1}, \frac{1}{\lambda}\left(e^{\lambda t}-1\right)\right), \beta_{n, \lambda}(x) \sim\left(\frac{\lambda\left(e^{t}-1\right)}{e^{\lambda t}-1}, \frac{1}{\lambda}\left(e^{\lambda t}-1\right)\right)$. Also, $(x)_{n, \lambda}$ is $\lambda$-Sheffer for $(1, t),(x)_{n, \lambda} \sim$ $(1, t)_{\lambda}$ and Sheffer for $\left(1, \frac{1}{\lambda}\left(e^{\lambda t}-1\right)\right),(x)_{n, \lambda} \sim\left(1, \frac{1}{\lambda}\left(e^{\lambda t}-1\right)\right)$. Let $\beta_{n, \lambda}(x)=\sum_{k=0}^{n} C_{n, k}(x)_{k, \lambda}$. Then it is obviously easier to compute the coefficients $C_{n, k}$ by viewing $\beta_{n, \lambda}(x)$ and $(x)_{n, \lambda}$ as $\lambda$-Sheffer sequences and using (20) than by viewing them as Sheffer sequences and using (22).

In this paper, we study the properties of degenerate poly-Bernoulli polynomial arising from degenerate polylogarithmic function and give some identities of those polynomials associated with special polynomials which are derived from the properties of $\lambda$-Sheffer sequences.

\section{Representations of degenerate poly-Bernoulli polynomials}

For $S_{n, \lambda}(x) \sim(g(t), f(t))_{\lambda},(n \geq 0)$, we have

$$
\begin{aligned}
\left\langle\frac{1}{g(\bar{f}(t))} e_{\lambda}^{x}(\bar{f}(t)) \mid(x)_{n, \lambda}\right\rangle_{\lambda} & =\sum_{k=0}^{\infty} S_{k, \lambda}(x) \frac{1}{k !}\left\langle t^{k} \mid(x)_{n, \lambda}\right\rangle_{\lambda} \\
& =S_{n, \lambda}(x), \quad(n \geq 0) .
\end{aligned}
$$


In (23), we note that

$$
\begin{aligned}
\left\langle\frac{1}{g(\bar{f}(t))} e_{\lambda}^{x}(\bar{f}(t)) \mid(x)_{n, \lambda}\right\rangle_{\lambda} & =\sum_{j=0}^{\infty} \frac{1}{j !}\left\langle\frac{1}{g(\bar{f}(t))}(\bar{f}(t))^{j} \mid(x)_{n, \lambda}\right\rangle_{\lambda}(x)_{j, \lambda} \\
& =\sum_{j=0}^{n} \frac{1}{j !}\left\langle\frac{1}{g(\bar{f}(t))}(\bar{f}(t))^{j} \mid(x)_{n, \lambda}\right\rangle_{\lambda}(x)_{j, \lambda} .
\end{aligned}
$$

Therefore, by (23) and (24), we obtain the following theorem.

Theorem 1 For $S_{n, \lambda}(x) \sim(g(t), f(t))_{\lambda}$, we have

$$
S_{n, \lambda}(x)=\sum_{j=0}^{n} \frac{1}{j !}\left\langle\frac{1}{g(\bar{f}(t))}(\bar{f}(t))^{j} \mid(x)_{n, \lambda}\right\rangle_{\lambda}(x)_{j, \lambda} .
$$

From (5) and (17), we have

$$
B_{n, \lambda}^{(k)}(x) \sim\left(\frac{e_{\lambda}(t)-1}{\operatorname{Li}_{k, \lambda}\left(1-e_{\lambda}(-t)\right)}, t\right)_{\lambda}
$$

By Theorem 1 applied to (25), we get the following corollary.

Corollary 2 For $n \geq 0$, we have

$$
B_{n, \lambda}^{(k)}(x)=\sum_{j=0}^{n}\left(\begin{array}{l}
n \\
j
\end{array}\right) B_{n-j, \lambda}^{(k)}(x)_{j, \lambda} .
$$

Here we remark that Corollary 2 can also be obtained by combining (15) and the first line of (27).

From (25), we note that

$$
\left(\frac{e_{\lambda}(t)-1}{\operatorname{Li}_{k, \lambda}\left(1-e_{\lambda}(-t)\right)}\right)_{\lambda} B_{n, \lambda}^{(k)}(x)=(x)_{n, \lambda} \sim(1, t)_{\lambda}
$$

By (26), and noting that $(x)_{n, \lambda}=\sum_{l=0}^{n} \sum_{j=0}^{l} S_{2, \lambda}(n, l) S_{1, \lambda}(l, j)(x)_{j, \lambda}$, we get

$$
\begin{aligned}
B_{n, \lambda}^{(k)}(x) & =\left(\frac{\operatorname{Li}_{k, \lambda}\left(1-e_{\lambda}(-t)\right)}{e_{\lambda}(t)-1}\right)_{\lambda}(x)_{n, \lambda} \\
& =\sum_{l=0}^{n} \sum_{j=0}^{l} S_{2, \lambda}(n, l) S_{1, \lambda}(l, j)\left(\frac{\operatorname{Li}_{k, \lambda}\left(1-e_{\lambda}(-t)\right)}{e_{\lambda}(t)-1}\right)_{\lambda}(x)_{j, \lambda} \\
& =\sum_{l=0}^{n} \sum_{j=0}^{l} S_{2, \lambda}(n, l) S_{1, \lambda}(l, j) \sum_{m=0}^{j} B_{m, \lambda}^{(k)} \frac{1}{m !}\left(t^{m}\right)_{\lambda}(x)_{j, \lambda} \\
& =\sum_{l=0}^{n} \sum_{j=0}^{l} \sum_{m=0}^{j}\left(\begin{array}{c}
j \\
m
\end{array}\right) S_{2, \lambda}(n, l) S_{1, \lambda}(l, j) B_{m, \lambda}^{(k)}(x)_{j-m, \lambda}
\end{aligned}
$$




$$
\begin{aligned}
& =\sum_{l=0}^{n} \sum_{m=0}^{l} \sum_{j=m}^{l}\left(\begin{array}{c}
j \\
m
\end{array}\right) S_{2, \lambda}(n, l) S_{1, \lambda}(l, j) B_{m, \lambda}^{(k)}(x)_{j-m, \lambda} \\
& =\sum_{l=0}^{n} \sum_{m=0}^{l} \sum_{j=0}^{l-m}\left(\begin{array}{c}
j+m \\
m
\end{array}\right) S_{2, \lambda}(n, l) S_{1, \lambda}(l, j+m) B_{m, \lambda}^{(k)}(x)_{j, \lambda} .
\end{aligned}
$$

Therefore, by (27), we obtain the following theorem.

Theorem 3 For $n \geq 0$, we have

$$
B_{n, \lambda}^{(k)}(x)=\sum_{l=0}^{n} \sum_{m=0}^{l} \sum_{j=0}^{l-m}\left(\begin{array}{c}
j+m \\
m
\end{array}\right) S_{2, \lambda}(n, l) S_{1, \lambda}(l, j+m) B_{m, \lambda}^{(k)}(x)_{j, \lambda} .
$$

Now, we observe that

$$
\begin{aligned}
& B_{n, \lambda}^{(k)}(y)=\left\langle\frac{\mathrm{Li}_{k, \lambda}\left(1-e_{\lambda}(-t)\right)}{e_{\lambda}(t)-1} e_{\lambda}^{y}(t) \mid(x)_{n, \lambda}\right\rangle_{\lambda} \\
& =\left\langle\frac{\operatorname{Li}_{k, \lambda}\left(1-e_{\lambda}(-t)\right)}{t} \mid\left(\frac{t}{e_{\lambda}(t)-1} e_{\lambda}^{y}(t)\right)_{\lambda}(x)_{n, \lambda}\right\rangle_{\lambda} \\
& =\sum_{l=0}^{n}\left(\begin{array}{l}
n \\
l
\end{array}\right) \beta_{l, \lambda}(y)\left\langle\frac{1}{t} \operatorname{Li}_{k, \lambda}\left(1-e_{\lambda}(-t)\right) \mid(x)_{n-l, \lambda}\right\rangle_{\lambda} \\
& =\sum_{l=0}^{n}\left(\begin{array}{l}
n \\
l
\end{array}\right) \beta_{l, \lambda}(y)\left\langle\frac{1}{t} \sum_{m=1}^{\infty} \frac{(-\lambda)^{m-1}(1)_{m, 1 / \lambda}}{m^{k-1}} \frac{(-1)^{m}}{m !}\left(e_{\lambda}(-t)-1\right)^{m} \mid(x)_{n-l, \lambda}\right\rangle_{\lambda} \\
& =\sum_{l=0}^{n}\left(\begin{array}{l}
n \\
l
\end{array}\right) \beta_{l, \lambda}(y)\left\langle\frac { 1 } { t } \sum _ { j = 1 } ^ { \infty } \left(\sum_{m=1}^{j} \frac{(-\lambda)^{m-1}(1)_{m, 1 / \lambda}}{m^{k-1}}(-1)^{j-m} S_{2, \lambda}(j, m) \frac{t^{j}}{j !}\left|(x)_{n-l, \lambda}\right\rangle_{\lambda}\right.\right. \\
& =\sum_{l=0}^{n}\left(\begin{array}{l}
n \\
l
\end{array}\right) \beta_{l, \lambda}(y) \sum_{j=0}^{\infty}\left(\sum_{m=1}^{j+1} \frac{(-\lambda)^{m-1}(1)_{m, 1 / \lambda}}{m^{k-1}}(-1)^{j+1-m} \frac{S_{2, \lambda}(j+1, m)}{(j+1) !}\right)\left\langle t^{j} \mid(x)_{n-l, \lambda}\right\rangle_{\lambda} \\
& =\sum_{l=0}^{n}\left(\begin{array}{l}
n \\
l
\end{array}\right) \beta_{l, \lambda}(y) \sum_{m=1}^{n-l+1} \frac{(-\lambda)^{m-1}(1)_{m, 1 / \lambda}}{m^{k-1}} \frac{(-1)^{n-l+1-m}}{(n-l+1) !} S_{2, \lambda}(n-l+1, m)(n-l) ! \\
& =\sum_{l=0}^{n} \sum_{m=1}^{n-l+1} \frac{(-1)^{n-l}\left(\begin{array}{c}
n \\
l
\end{array}\right) \lambda^{m-1}}{(n-l+1) m^{k-1}}(1)_{m, 1 / \lambda} S_{2, \lambda}(n-l+1, m) \beta_{l, \lambda}(y)
\end{aligned}
$$

Therefore, by (28), we obtain the following theorem.

Theorem 4 For $n \geq 0$, we have

$$
B_{n, \lambda}^{(k)}(x)=\sum_{l=0}^{n} \sum_{m=1}^{n-l+1} \frac{(-1)^{n-l}\left(\begin{array}{c}
n \\
l
\end{array}\right) \lambda^{m-1}}{(n-l+1) m^{k-1}}(1)_{m, 1 / \lambda} S_{2, \lambda}(n-l+1, m) \beta_{l, \lambda}(x) .
$$

By (6) and (17), we note that

$$
\beta_{n, \lambda}^{(s)}(x) \sim\left(\frac{\left(e_{\lambda}(t)-1\right)^{s}}{t^{s}}, t\right)_{\lambda}
$$


From (19), (25), and (29), we have

$$
B_{n, \lambda}^{(k)}(x)=\sum_{m=0}^{n} C_{n, m} \beta_{m, \lambda}^{(s)}(x),
$$

where

$$
\begin{aligned}
C_{n, m} & =\frac{1}{m !}\left\langle\frac{\mathrm{Li}_{k, \lambda}\left(1-e_{\lambda}(-t)\right)}{e_{\lambda}(t)-1} \frac{\left(e_{\lambda}(t)-1\right)^{s}}{t^{s}} t^{m} \mid(x)_{n, \lambda}\right\rangle_{\lambda} \\
& =\left(\begin{array}{c}
n \\
m
\end{array}\right)\left\langle\frac{\mathrm{Li}_{k, \lambda}\left(1-e_{\lambda}(-t)\right)}{e_{\lambda}(t)-1} \frac{\left(e_{\lambda}(t)-1\right)^{s}}{t^{s}} \mid(x)_{n-m, \lambda}\right\rangle_{\lambda} \\
& =\left(\begin{array}{c}
n \\
m
\end{array}\right) \sum_{l=0}^{n-m} \frac{S_{2, \lambda}(l+s, s)}{\left(\begin{array}{c}
l+s \\
s
\end{array}\right) l !}\left\langle\frac{\mathrm{Li}_{k, \lambda}\left(1-e_{\lambda}(-t)\right)}{e_{\lambda}(t)-1} t^{l} \mid(x)_{n-m, \lambda}\right\rangle_{\lambda} \\
& =\left(\begin{array}{c}
n \\
m
\end{array}\right) \sum_{l=0}^{n-m} \frac{S_{2, \lambda}(l+s, s)}{\left(\begin{array}{c}
l+s \\
s
\end{array}\right)}\left(\begin{array}{c}
n-m \\
l
\end{array}\right)\left\langle\frac{\mathrm{Li}_{k, \lambda}\left(1-e_{\lambda}(-t)\right)}{e_{\lambda}(t)-1} \mid(x)_{n-m-l, \lambda}\right\rangle_{\lambda} \\
& =\left(\begin{array}{c}
n \\
m
\end{array}\right) \sum_{l=0}^{n-m} \frac{S_{2, \lambda}(l+s, s)}{\left(\begin{array}{c}
l+s \\
s
\end{array}\right)}\left(\begin{array}{c}
n-m \\
l
\end{array}\right) B_{n-m-l, \lambda}^{(k)} .
\end{aligned}
$$

Therefore, by (30) and (31), we obtain the following theorem.

Theorem 5 For $n \geq 0$, we have

$$
B_{n, \lambda}^{(k)}(x)=\sum_{m=0}^{n}\left(\begin{array}{c}
n \\
m
\end{array}\right)\left(\sum_{l=0}^{n-m} \frac{\left(\begin{array}{c}
n-m \\
l
\end{array}\right)}{\left(\begin{array}{c}
l+s \\
s
\end{array}\right)} S_{2, \lambda}(l+s, s) B_{n-m-l, \lambda}^{(k)}\right) \beta_{m, \lambda}^{(s)}(x) .
$$

For $n \geq 0$, we let

$$
\mathbb{P}_{n}=\{p(x) \in \mathbb{C}[x] \mid \operatorname{deg} p(x) \leq n\}
$$

Then $\mathbb{P}_{n}$ is an $(n+1)$-dimensional vector space over $\mathbb{C}$.

From (11), we note that $d_{n, \lambda}(x) \sim\left((1-t) e_{\lambda}(t), t\right)_{\lambda},(n \geq 0)$. For $p(x) \in \mathbb{P}_{n}$, we let

$$
p(x)=\sum_{l=0}^{n} C_{l} d_{l, \lambda}(x)
$$

By (16), we have

$$
\begin{aligned}
\left\langle(1-t) e_{\lambda}(t) t^{m} \mid p(x)\right\rangle_{\lambda} & =\sum_{l=0}^{n} C_{l}\left\langle(1-t) e_{\lambda}(t) t^{m} \mid d_{l, \lambda}(x)\right\rangle_{\lambda} \\
& =\sum_{l=0}^{n} C_{l} m ! \delta_{m, l}=C_{m} m !,
\end{aligned}
$$

where $0 \leq m \leq n$.

Therefore, by (32) and (33), we obtain the following theorem. 
Theorem 6 For $p(x) \in \mathbb{P}_{n}$, we have

$$
p(x)=\sum_{l=0}^{n} C_{l} d_{l, \lambda}(x),
$$

where

$$
C_{l}=\frac{1}{l !}\left\langle(1-t) e_{\lambda} t^{l} \mid p(x)\right\rangle_{\lambda}
$$

Let $p(x)=B_{n, \lambda}^{(k)}(x) \in \mathbb{P}_{n}$. Then we have

$$
B_{n, \lambda}^{(k)}(x)=\sum_{l=0}^{n} C_{l} d_{l, \lambda}(x)
$$

where

$$
\begin{aligned}
C_{l} & =\frac{1}{l !}\left\langle(1-t) e_{\lambda}(t) t^{l} \mid B_{n, \lambda}^{(k)}(x)\right\rangle_{\lambda} \\
& =\left(\begin{array}{c}
n \\
l
\end{array}\right)\left\langle(1-t) e_{\lambda}(t) \mid B_{n-l, \lambda}^{(k)}(x)\right\rangle_{\lambda} \\
& =\left(\begin{array}{c}
n \\
l
\end{array}\right)\left\langle(1-t) \mid B_{n-l, \lambda}^{(k)}(x+1)\right\rangle_{\lambda} \\
& =\left(\begin{array}{c}
n \\
l
\end{array}\right)\left\langle 1 \mid B_{n-l, \lambda}^{(k)}(x+1)\right\rangle_{\lambda}-\left(\begin{array}{c}
n \\
l
\end{array}\right)(n-l)\left\langle 1 \mid B_{n-l-1, \lambda}^{(k)}(x+1)\right\rangle_{\lambda} \\
& =\left(\begin{array}{c}
n \\
l
\end{array}\right) B_{n-l, \lambda}^{(k)}-n\left(\begin{array}{c}
n-1 \\
l
\end{array}\right) B_{n-l-1, \lambda}^{(k)}(1) .
\end{aligned}
$$

Thus, by (34) and (35), we get

$$
B_{n, \lambda}^{(k)}(x)=\sum_{l=0}^{n}\left(\left(\begin{array}{c}
n \\
l
\end{array}\right) B_{n-l, \lambda}^{(k)}-n\left(\begin{array}{c}
n-1 \\
l
\end{array}\right) B_{n-l-1, \lambda}^{(k)}\right) d_{l, \lambda}(x) .
$$

From (11), we note that $d_{n, r}^{(r)}(x) \sim\left((1-t)^{r} e_{\lambda}(t), t\right)_{\lambda}$.

Let us assume that

$$
p(x)=\sum_{m=0}^{n} C_{m}^{(r)} d_{m, \lambda}^{(r)}(x) \in \mathbb{P}_{n}
$$

Then, by (16), we get

$$
\begin{aligned}
\left\langle(1-t)^{r} e_{\lambda}(t) t^{m} \mid p(x)\right\rangle_{\lambda} & =\sum_{l=0}^{n} C_{l}^{(r)}\left\langle(1-t)^{r} e_{\lambda}(t) t^{m} \mid d_{l, \lambda}^{(r)}(x)\right\rangle_{\lambda} \\
& =m ! C_{m}^{(r)} \quad(0 \leq m \leq n) .
\end{aligned}
$$

Therefore, by (37) and (38), we obtain the following theorem. 
Theorem 7 For $n \geq 0$, we have

$$
p(x)=\sum_{m=0}^{n} C_{m}^{(r)} d_{m, \lambda}^{(r)}(x)
$$

where

$$
C_{m}^{(r)}=\frac{1}{m !}\left\langle(1-t)^{r} e_{\lambda}(t) t^{m} \mid p(x)\right\rangle_{\lambda}
$$

We let

$$
d_{n, \lambda}(x)=\sum_{m=0}^{n} C_{m}^{(r)} d_{m, \lambda}^{(r)}(x)
$$

where

$$
\begin{aligned}
C_{m}^{(r)} & =\frac{1}{m !}\left\langle(1-t)^{r} e_{\lambda}(t) t^{m} \mid d_{n, \lambda}(x)\right\rangle_{\lambda} \\
& =\left(\begin{array}{c}
n \\
m
\end{array}\right)\left\langle(1-t)^{r} \mid d_{n-m, \lambda}(x+1)\right\rangle_{\lambda} \\
& =\left(\begin{array}{c}
n \\
m
\end{array}\right) \sum_{j=0}^{r}\left(\begin{array}{l}
r \\
j
\end{array}\right)(-1)^{j}(n-m)_{j}\left\langle 1 \mid d_{n-m-j, \lambda}(x+1)\right\rangle_{\lambda} \\
& =\left(\begin{array}{c}
n \\
m
\end{array}\right) \sum_{j=0}^{r}\left(\begin{array}{c}
r \\
j
\end{array}\right)\left(\begin{array}{c}
n-m \\
j
\end{array}\right)(-1)^{j} d_{n-m-j, \lambda}(1) j ! .
\end{aligned}
$$

By (39) and (40), we get

$$
d_{n, \lambda}(x)=\sum_{m=0}^{n}\left(\begin{array}{l}
n \\
m
\end{array}\right)\left(\sum_{j=0}^{r}\left(\begin{array}{c}
r \\
j
\end{array}\right)\left(\begin{array}{c}
n-m \\
j
\end{array}\right) j !(-1)^{j} d_{n-m-j, \lambda}(1)\right) d_{m, \lambda}^{(r)}(x), \quad(n \geq 0) .
$$

Let us take $p(x)=B_{n, \lambda}^{(k)}(x) \in \mathbb{P}_{n},(n \geq 0)$. Then, by Theorem 7 , we get

$$
B_{n, \lambda}^{(k)}(x)=\sum_{m=0}^{n} C_{m}^{(r)} d_{m, \lambda}^{(r)}(x), \quad(n \geq 0)
$$

where

$$
\begin{aligned}
C_{m}^{(r)} & =\frac{1}{m !}\left\langle(1-t)^{r} e_{\lambda}(t) t^{m} \mid B_{n, \lambda}^{(k)}(x)\right\rangle_{\lambda} \\
& =\left(\begin{array}{c}
n \\
m
\end{array}\right)\left\langle(1-t)^{r} \mid B_{n-m, \lambda}^{(k)}(x+1)\right\rangle_{\lambda} \\
& =\left(\begin{array}{c}
n \\
m
\end{array}\right) \sum_{j=0}^{r}\left(\begin{array}{c}
r \\
j
\end{array}\right)(-1)^{j}\left(\begin{array}{c}
n-m \\
j
\end{array}\right) j !\left(1\left|B_{n-m-j}^{(k)}(x+1)\right\rangle_{\lambda}\right. \\
& =\left(\begin{array}{c}
n \\
m
\end{array}\right) \sum_{j=0}^{r}\left(\begin{array}{c}
r \\
j
\end{array}\right)(-1)^{j}\left(\begin{array}{c}
n-m \\
j
\end{array}\right) j ! B_{n-m-j, \lambda}^{(k)}(1) .
\end{aligned}
$$

Therefore, by (42) and (43), we obtain the following theorem. 
Theorem 8 For $n \geq 0, k \in \mathbb{Z}$, and $r \in \mathbb{N}$, we have

$$
B_{n, \lambda}^{(k)}(x)=\sum_{m=0}^{n}\left(\begin{array}{l}
n \\
m
\end{array}\right)\left(\sum_{j=0}^{r}\left(\begin{array}{l}
r \\
j
\end{array}\right)(-1)^{j}\left(\begin{array}{c}
n-m \\
j
\end{array}\right) j ! B_{n-m-j, \lambda}^{(k)}(1)\right) d_{m, \lambda}^{(r)}(x) .
$$

\section{Conclusion}

The umbral calculus had been laid as a rigorous foundation by Rota and is based on linear functionals, differential operators, and Sheffer sequences. Here, for an invertible series $g(t)$ and a delta series $f(t), S_{n}(x)$ is the Sheffer sequence for $(g(t), f(t))$ if and only if

$$
\frac{1}{g(\bar{f}(t))} e^{x \bar{f}(t)}=\sum_{n=0}^{\infty} S_{n}(x) \frac{t^{n}}{n !}
$$

Recently, the ' $\lambda$-umbral calculus' was developed by the motivation that what if the usual exponential function appearing in (44) is replaced with the degenerate exponential functions in (2). This question led us to the introduction of the concepts like $\lambda$-linear functionals, $\lambda$-differential operators, and $\lambda$-Sheffer sequences. In fact, for $g(t)$ and $f(t)$ as before, the sequence $S_{n, \lambda}(x)$ is the $\lambda$-Sheffer sequence for $(g(t), f(t))$ if and only if

$$
\frac{1}{g(\bar{f}(t))} e_{\lambda}^{x}(\bar{f}(t))=\sum_{n=0}^{\infty} S_{n, \lambda}(x) \frac{t^{n}}{n !} .
$$

We noted that the $\lambda$-umbral calculus has some advantage over the traditional umbral calculus when dealing with $\lambda$-Sheffer sequences. This was illustrated with the problem of representing the degenerate Bernoulli polynomial in terms of the degenerate falling factorials. Moreover, the introduction of the $\lambda$-umbral calculus is natural in view of the recent regained interests of many mathematicians in the study of degenerate versions of some special polynomials and numbers, which was initiated by Carlitz.

In this paper, in order to study the degenerate poly-Bernoulli polynomials, which is a $\lambda$-Sheffer sequence, we used three different formulas, namely a formula about representing a $\lambda$-Sheffer sequence by another, a formula coming from the generating functions of $\lambda$-Sheffer sequences, and a formula arising from the definitions for $\lambda$-Sheffer sequences. Then we represented, among other things, the degenerate poly-Bernoulli polynomials by higher-order degenerate Bernoulli polynomials and also by higher-order degenerate derangement polynomials.

It is one of our future projects to continue to investigate the degenerate special numbers and polynomials by using the recently developed $\lambda$-umbral calculus.

\section{Acknowledgements}

The authors would like to thank the reviewers for their detailed comments and suggestions that helped improve the original manuscript in its present form. Also, they thank Jangjeon Research Institute for Mathematical Sciences for the support of this research.

Funding

The first author has been conducted by the Research Grant of Kwangwoon University in 2021. And the third author was supported by the National Research Foundation of Korea (NRF)grant funded by the Korea government (No. 2020R1F1A1A01071564) 
Ethics approval and consent to participate

All authors reveal that there is no ethical problem in the production of this paper.

\section{Competing interests}

The authors declare that they have no competing interests.

Consent for publication

All authors want to publish this paper in this journal.

\section{Authors' contributions}

TK and DSK conceived of the framework and structured the whole paper; TK and DSK wrote the paper; JK and HL checked the results of the paper and typed the paper; DSK and TK completed the revision of the article. All authors have read and agreed to the published version of the manuscript.

\section{Author details}

'School of Science, Xian Technological University, Xi'an 710021 Shaan, China. ${ }^{2}$ Department of Mathematics, Kwangwoon University, Seoul 139-701, Republic of Korea. ${ }^{3}$ Department of Mathematics, Sogang University, Seoul 121-742, Republic of Korea. ${ }^{4}$ Department of Mathematics Education and ERI, Gyeongsang National University, Jinju 660-701, Republic of Korea.

\section{Publisher's Note}

Springer Nature remains neutral with regard to jurisdictional claims in published maps and institutional affiliations.

\section{Received: 11 December 2020 Accepted: 18 March 2021 Published online: 26 March 2021}

\section{References}

1. Bayad, A., Hamahata, Y.: Polylogarithms and poly-Bernoulli polynomials. Kyushu J. Math. 65(1), 15-24 (2011)

2. Carlitz, L.: Degenerate Stirling, Bernoulli and Eulerian numbers. Util. Math. 15, 51-88 (1979)

3. Dere, R., Simsek, Y.: Applications of umbral algebra to some special polynomials. Adv. Stud. Contemp. Math. (Kyungshang) 22(3), 433-438 (2012)

4. Dere, R., Simsek, Y.: Hermite base Bernoulli type polynomials on the umbral algebra. Russ. J. Math. Phys. 22(1), 1-5 (2015)

5. Dere, R., Simsek, Y., Srivastava, H.M.: A unified presentation of three families of generalized Apostol type polynomials based upon the theory of the umbral calculus and the umbral algebra. J. Number Theory 133, 3245-3263 (2013)

6. Gzyl, H.: Canonical transformations, umbral calculus, and orthogonal theory. J. Math. Anal. Appl. 111(2), 547-558 (1985)

7. Kholodov, A.N.: The umbral calculus and orthogonal polynomials. Acta Appl. Math. 19(1), 1-54 (1990)

8. Kim, D.S., Kim, T.: A note on polyexponential and unipoly functions. Russ. J. Math. Phys. 26(1), 40-49 (2019)

9. Kim, D.S., Kim, T.: A note on a new type of degenerate Bernoulli numbers. Russ. J. Math. Phys. 27(2), 227-235 (2020)

10. Kim, D.S., Kim, T.: Sheffer sequences and $\lambda$-Sheffer sequences. J. Math. Anal. Appl. 493(1), 124521 (2021)

11. Kim, T.: A note on degenerate Stirling polynomials of the second kind. Proc. Jangjeon Math. Soc. 20(3), 319-331 (2017)

12. Kim, T., Kim, D.S.: Some identities on derangement and degenerate derangement polynomials. In: Advances in Mathematical Inequalities and Applications. Trends Math., pp. 265-277. Springer, Singapore (2018)

13. Kim, T., Kim, D.S.: Note on the degenerate gamma function. Russ. J. Math. Phys. 27(3), 352-358 (2020)

14. Kim, T., Kim, D.S.: Degenerate polyexponential functions and degenerate Bell polynomials. J. Math. Anal. Appl. 487(2), $124017(2020)$

15. Kim, T., Kim, D.S., Dolgy, D.V., Kwon, J.: Some identities of derangement numbers. Proc. Jangjeon Math. Soc. 21(1), 125-141 (2018)

16. Kim, T., Kim, D.S., Jang, G.-W., Kwon, J.: A note on some identities of derangement polynomials. J. Inequal. Appl. 2018, 40 (2018)

17. Kim, T., Kim, D.S., Kim, H.-Y., Lee, H., Jang, L.-C.: Degenerate poly-Bernoulli polynomials arising from degenerate polylogarithm. Adv. Differ. Equ. 2020, 444 (2020)

18. Kim, T., Kim, D.S., Kwon, H.-I., Jang, L.-C.: Fourier series of sums of products of $r$-derangement functions. J. Nonlinear Sci. Appl. 11(4), 575-590 (2018)

19. Kim, T., Kim, D.S., Lee, H., Jang, L.-C.: A note on degenerate derangement polynomials and numbers. https://arxiv.org/abs/2011.08535

20. Komatsu, T., Simsek, Y.: Identities related to the Stirling numbers and modified Apostol-type numbers on umbral calculus. Miskolc Math. Notes 18(2), 905-916 (2017)

21. Lee, D.S., Kim, H.K.: On the new type of degenerate poly-Genocchi numbers and polynomials. Adv. Differ. Equ. 2020, $431(2020)$

22. Lewin, L.: Polylogarithms and Associated Functions. North-Holland, Amsterdam (1981)

23. Ray, N.: Extensions of umbral calculus: penumbral coalgebras and generalised Bernoulli numbers. Adv. Math. 61(1), 49-100 (1986)

24. Roman, S.: The Umbral Calculus. Pure and Applied Mathematics, vol. 111. Academic Press, New York (1984)

25. Rota, G.-C., Taylor, B.D.: The classical umbral calculus. SIAM J. Math. Anal. 25(2), 694-711 (1994)

26. Ueno, K.: General power umbral calculus in several variables. J. Pure Appl. Algebra 59(3), 299-308 (1989)

27. Wilson, B.G., Rogers, F.J.: Umbral calculus and the theory of multispecies nonideal gases. Physica A 139(2-3), 359-386 (1986) 